\title{
Feedback from the Science Café from the Sixth European Bioanalysis Forum Young Scientist Symposium
}

\author{
Salvatore Calogero ${ }^{1}$, Lisa Delahaye ${ }^{2}$, Cara Gunning ${ }^{3}$, Terence Hetzel ${ }^{4}$, Tessa McDonald ${ }^{5}$, \\ Coral Munday ${ }^{6}$, Michele Protti ${ }^{7}$, Mathias Salger ${ }^{8}$, Laura Schlicksupp ${ }^{9}$, Bas Sleumer ${ }^{10}$, \\ Connor Walker ${ }^{11}$, Adam Williams ${ }^{12}$, Robin Wördenweber ${ }^{13}$ \& Philip Timmerman*, 14 \\ ${ }^{1}$ Bioanalytical R\&D, F. Hoffmann-La Roche Ltd, Regulated Bioanalysis, Basel 4070, Switzerland, employed by Swiss BioQuant, \\ Biochemical analysis, Reinach (BL) 4153 , Switzerland during the meeting \& writing of this manuscript \\ ${ }^{2}$ Laboratory of Toxicology, Ghent University, Ghent 9000, Belgium \\ ${ }^{3}$ Immunobiology, Charles River Laboratories, Edinburgh EH33 2NE, UK \\ ${ }^{4}$ Bioanalysis, Bayer AG, Wuppertal 42096, Germany \\ ${ }^{5}$ Immunoassay, Unilabs York Bioanalytical Solutions, York YO26 6QR, UK \\ ${ }^{6}$ LGC, Drug Development Solutions, Fordham CB7 5WW, UK \\ ${ }^{7}$ Department of Pharmacy \& Biotechnology (FaBiT), Alma Mater Studiorum - University of Bologna, Research group of \\ Pharmaco-Toxicological Analysis (PTA Lab), Bologna 40127, Italy \\ ${ }^{8}$ Bioanalysis, Nuvisan GmbH, Neu-Ulm 89231, Germany \\ ${ }^{9}$ AbbVie Deutschland GmbH \& Co. KG, DMPK-BA Bioanalysis, Ludwigshafen (Rhein) 67061, Germany \\ ${ }^{10}$ PRA Health Sciences, Bioanalytical Laboratory, Assen 9407 TK, The Netherlands \\ ${ }^{11}$ Arcinova, Bioanalytical Services, Alnwick NE66 2DH, UK \\ ${ }^{12}$ Clinical Pharmacology \& Safety Sciences, AstraZeneca, Cambridge CB21 6GH, UK \\ ${ }^{13}$ A\&M Labor, Bergheim 50126, Germany \\ ${ }^{14}$ Philip Timmerman, European Bioanalysis Forum vzW, Brussels 1000, Belgium \\ *Author for correspondence: chair@e-b-f.eu
}

The 6th Young Scientist Symposium, a meeting organized by young scientists for young scientists under the umbrella of the European Bioanalysis Forum vzw and in collaboration with the Universities of Bologna and Ghent, included a variety of interesting presentations on cutting edge bioanalytical science and processes. Integrated in the meeting, an interactive round table session, the Science Café, discussed the challenges related to sustainability for bioanalytical lab activities. This manuscript reflects conclusions from these discussions. They can provide our community a compass for future business practices to embrace more sustainable laboratory activities considerate of smarter use of a wide array of resources and laboratory tools, resulting in increased wellbeing for our next generations and our planet.

First draft submitted: 7 January 2021; Accepted for publication: 18 January 2021; Published online:

4 February 2021

Keywords: EBF • sustainability $\bullet$ young scientist

Since 2014, the European Bioanalysis Forum vzw (EBF) organizes an annual Young Scientist Symposium (YSS). This meeting, which is part of EBF's Mission statement [1] aims at providing scientific and cross-company collaboration opportunities for young scientists. As a nonsponsored, nonprofit meeting, the YSS is unique in its format, in that it is organized by young scientists for young scientists. At the same time, meeting attendance is reserved for new talent in the bioanalytical laboratory, creating an environment that is not biased, influenced or overshadowed by senior management/experts leading the discussions. The collaboration of university laboratories (i.e., University of Bologna and University of Ghent, Belgium), builds the necessary bridge to academic labs.

Organizing the meeting in 2020 was not easy. This is an understatement. The program was ready to go when the coronavirus pandemic [2] hit the world midship. We had to redesign the meeting from a face-to-face event (originally scheduled in March) into a mixed virtual event and postpone until we had a better view on the community and industry damage caused by the virus. Rescheduling also involved keeping the engagement and commitment of all presenters and delegates to travel with us in time and space. Finally, the meeting would run end of September of 
2020 , in cyberspace. Fortunately, we succeeded to still safely have a limited face-to-face connection in some regions, regional regulations and safety precautions permitting. In this way, and under strict safety guidelines, small groups of delegates could participate to the online event from one (spacious) meeting room.

The scientific program was strong and diverse. All details on the program and the majority of the presentations (as PDF) can be found on the EBF website [3]. And, with almost 100 delegates registered, it was the best attended YSS to date.

\section{The Science Café}

An important session in each YSS is the Science Café. This is an interactive format, designed in 2018, where the delegates reflect and discuss around strategic themes related to their science, processes or regulations. In previous Science Cafés, themes, such as regulatory requirements on incurred sample reproducibility (ISR), automation, biomarkers or the advantages and challenges of lean approaches to validation were discussed $[4,5]$. This year, and not coincidentally related to the strong focus of environmental impact of human activity which made news headlines when the coronavirus was not yet on the horizon, the young scientist community chose 'sustainability' as this year's theme.

In this manuscript, we will not discuss the plenary sessions, but we will give feedback of the discussions and the ideas or solutions suggested during the Science Café. The Science Café was held in the morning of the 2nd day, partially as face-to-face roundtable in four different locations, and partially online in six breakout sessions in cyberspace.

To prepare the delegates, a survey of seven questions, all with the theme of sustainability in bioanalysis, was sent out a few weeks prior to the event. As part of this survey, the delegates were invited to also read reference papers for inspiration on the subjects [6-10].

Responses received were used to drive the conversation during roundtable discussion.

\section{Question 1: in your opinion, is sustainable regulated bioanalysis possible?}

As the topic of the Science Café was sustainability in regulated bioanalysis, the starting point of the discussion was whether, in the opinion of the delegates, sustainability is achievable within such an environment.

Sustainability is rather a generic definition. Therefore, it is not easy to answer the question exhaustively. The vast majority of the Young Scientists agreed to the fact that it is possible to reduce the environmental footprint of bioanalytical activities, however, they acknowledge some restrictions. In particular, the biggest concern is relative to the fact that, in the near future it is difficult to see an alternative to single-use plastic materials for the reduction of sample contamination (which is paramount with the exponential increase of sensitivity of modern instrumentations).

Many delegates pointed out the fact that, at least with current material technologies, there is no alternative to single-use plastic pipette tips. Furthermore, it is not always possible to recycle this type of waste, since it would mean introducing dangerous materials, such as biological and (radio) chemical waste, into recycling stations which may not be equipped to handle this kind of waste.

An additional concern is that, in a regulated environment, regulatory guidelines might represent a restriction in the implementation of more ecological processes.

Last but not least, implementation of new processes implies a start-up cost, in which many stakeholders are not willing to invest because they do not see an immediate return. An example of this was the switch from paper data recording to electronic. One or two delegates confirmed that their companies had made this switch already, others stated that paper was still mostly being used.

\section{Question 2: within your team, have there been discussions of investing resources to improve sustainability?}

The first question set the stage for the second part of the discussion: is sustainability a topic within our laboratories?

Most of the delegates confirmed that the topic of sustainability is present in their teams and/or companies, however with different sensibilities.

Indeed, the problem of single-use materials is surely something that deserves in-depth reflection due to the impact that this waste has, for example, on our atmosphere in terms of $\mathrm{CO}_{2}$ and toxic products generated upon their production and disposal, in the ecosystem in terms of microplastics which enter into the food chain and ultimately impact health. 
In agreement to this assumption, most of the interviewed scientists confirmed that the discussion regarding the eco-friendly improvement in their respective laboratories takes mostly the form of reducing sample volumes, miniaturizing the workflows and reducing/recycling the waste when possible. Validation of higher temperatures for deep freezers, from -80 to $-65^{\circ} \mathrm{C}$ for example, is also something that some laboratories are considering.

\section{Question 3: have you seen a difference in efficiency/productivity since the pandemic, how do you} think it has affected sustainability in your laboratory?

"Coronavirus", "COVID-19" and "pandemic" are among the most searched terms in Google for 2020 for a reason [11]. This unprecedented situation, in recent times at least, had an impact on human life like nothing has since World War II.

The third question of the Science Café revolved around the influence that the COVID-19 pandemic had on delegate's laboratories in regard to sustainability.

Probably due to the fragmented approach by different countries in terms of countermeasures employed to fight the virus and the different capabilities that every company has to react to such an event, this was the question with the lowest degree of agreement among the delegates.

Indeed, while many noticed no difference in terms of sustainability, others have seen improvement, while some others believe the impact was negative.

People who thought the pandemic had a positive impact on sustainability in their working places mentioned the fact that implementation of home office helped to reduce the $\mathrm{CO}_{2}$ footprint on the environment due to the reduced number of cars on the streets because less people are commuting. On the other hand, many delegates believe that this reduction in carbon footprint was negated by the fact of surplus ordering and delivery, with related increase in transportation. Furthermore, the additional cleaning and safety precautions generated more waste, for example, by only offering take-away food in canteens with single-use plastics. In any case, most of these examples seem to be only indirectly related to the bioanalysis.

Some even found a silver lining to the disruption caused by the pandemic. This came in the form of an opportunity to re-think methods and processes, allowing for increased optimization and the consideration of alternative approaches. It should be said that some optimizations/adoption of alternatives might even be a necessity in order to continue analysis with current supply chain issues, as well as comply with social distancing guidelines.

\section{Question 4: are your colleagues open to optimizing methods/working process in the laboratory with consideration to sustainability? If so, what approaches do you take?}

The introduction of a new process always requires effort and additional work at first. All delegates agreed that collaboration and an open mindset among the team is fundamental to achieve a good result.

As demonstrated by the answers during the survey and the general trend of the discussion, it is clear that young scientists are quite happy to implement procedures which can optimize the work-flow and, at the same time, increase the sustainability of their work. However, one of the major problems seems to be the "we have always done it that way" mindset.

To overcome the issue, it was suggested that open dialogue and targeted training may have a positive impact on people's approach to the topic. With a view that today's junior team members will be tomorrow's leaders, training and a positive attitude toward sustainability will impact on future bioanalysis activities. Not forgetting momentum for change can be driven by sheer numbers if the will is great enough.

\section{Question 5: how can we achieve improved waste management?}

In practice, what are the concrete procedures that could help reduce the amount of waste and/or matrix consumption?

Coordination between different operators (or even different teams) to share common reagents seems to be the most applied and effective practice to reduce the amount of waste.

For the reduction of matrix usage, aligned with industry's desire to capitalize on the principles of the 3Rs (replacement, reduction and refinement), already during the method development phase, it is crucial to have an idea of the optimal assay range to develop methods which are not unnecessarily sensitive. In this way, higher minimal required dilution (MRDs) could be used and, also, it can be avoided that too many study samples need a dilution (with matrix) to fit into the detection range of the assay. 
The in-depth understanding of the instrument working cycles can be the key to better optimization of their usage and, consequently, reduced waste.

Some of the delegates also suggested the establishment of relationships with suppliers for the return of particular packaging or containers that could be re-used. During the discussions it became clear that the return of plastic waste by the suppliers can also be restricted to countries, however the delegates felt that getting the suppliers, contractors and so fourth, involved in the conversation could move suppliers to act and, therefore, ultimately improve waste management.

Question 6: which processes have you already implemented within your team to save resources? The delegates were asked what processes, if any, are already in place within their teams in order to save resources.

Although, as mentioned before, the implementation of new processes is often a slow process and there is a variety of suitable measures that can be taken by all laboratories with relatively low investment of time and resources. The majority of the answers confirmed this, indeed most of the strategies already used are simple but efficient. For example, donate old equipment to academia or schools instead of disposing of it to help students and save $\mathrm{CO}_{2}$.

Elementary gestures, like the refining of the dilution calculations, can lead to the reduction of reagent and matrix used during experiments. At the same time, optimizing the method design and planning weekly work for efficiencies could be a strategy to induce laboratory technicians to share common reagents and reduce waste. Where possible, re-test of materials in order to prolong shelf-life is a strategy that many teams are using.

The lowest common denominator of all the above is that those measures are not only reducing the environmental impact, but also saving money.

However, there is another type of process that the delegates mentioned, which requires an investment and is specific to the type of bioanalysis: implementation of micro-sampling, re-using of columns (if possible) or switching to new technologies (e.g., ultra [high] performance liquid chromatography [UPLC] vs HPLC or electrochemiluminescence immunoassay [ECLIA] vs ELISA).

\section{Question 7: do you think you should/could be doing more?}

It is easier to imagine what can be done to improve a process, when this action is meant to be taken by someone else. It is more difficult, instead, to realize what each of us could or should do. The answer to such a question is even more difficult when the audience who should answer is composed by young scientists who typically do not have a management role and do not have high decisional power. Probably, for this reason most of the comments of the delegates focused on the fact that what we, young scientists, should increase the involvement of our co-workers and create awareness of sustainability and inform on the true impact of our activities on the environment. For example, explaining to colleagues that to generate $100 \mathrm{ml}$ of mouse serum requires about 200 mice, could be a trigger to let them know the impact of what we are doing and stimulate 3Rs discussions.

The biggest challenge seems to be the "we have always done it that way" mentality. Bringing scientific evidence to support the thesis that new procedures, which are also more sustainable, are indeed better than the old-fashioned ones is the key to change the forma mentis.

On top of this, the easiest thing to start with is to provide an example, even for simple gestures, like switching off the lights before leaving, reducing the heating by 1 degree or increasing recycling.

\section{Summary/conclusion}

Overall, the consensus among the young scientists in this year's symposium was that many believed they could and maybe should be doing more. The change would not be easy however: similar concerns were voiced on the challenges that must be overcome to make bioanalysis more sustainable, for example, additional costs and planning for implementation, willingness of others, single use plastic waste and not being in a position to affect change within their organization.

In contrast with these concerns, most had a positive outlook and a willingness to improve their methods and habits. Many suggestions were provided on how we can achieve sustainability, including efficient method design and proper training, better communication and investment into smarter waste management. These suggestions are the first steps in overcoming the concerns of the viability of implementing sustainable practices. In regards to many feeling they were not in a position to drive change, YSS delegates were reminded during the feedback presentation that one day they would be in a position to make change, and that the journey to that goal began at the EBF YSS, where they have begun a dialogue with future leaders of the field. 
Some even showed more optimism, even finding a silver lining with the pandemic, believing that it has given a unique opportunity to rethink and challenge the "we have always done it that way" culture present in organizations. Another comment even went so far as to state that making bioanalysis more sustainable increases the value of work produced, and that as a scientific community, it is a goal we should strive to achieve.

One piece of feedback that stood out, was actually a question for the YSS. That question was "what were the EBF YSS doing in regards to sustainable bioanalysis?" The answer was the feedback presentation and this very paper, it is the beginning of a conversation to promote discussion and interest in the research and implementation of more sustainable practices in regulated bioanalysis.

With the feedback presented in this paper, the thoughts and feelings of the next generation of scientists and young scientists on sustainability in bioanalysis are made clear. The hope is with this information in hand, many will now engage with, or even initiate themselves, a dialogue in their organizations on researching and implementing sustainable practices in regulated bioanalysis. And, the YSS team has made the promise to evaluate actions taken on sustainability as part of the 7th YSS, which is scheduled for 10-11 June 2021, again in cyberspace.

Regardless of age, experience, role we can all do our part to help make regulated bioanalysis more sustainable, together we can make sustainable bioanalysis a reality, together we can be the change.

\section{Acknowledgments}

The authors thanked C Fox and H Schoeneberger for their contribution during the early planning phase of the 6th YSS and the Science Café. The EBF vzw would like to thank AbbVie, Arcinova, Boehringer Ingelheim, Charles River, Covance, Merck KGaA and PRA-HS for their support as patrons of the 6th YSS.

\section{Disclaimer}

The views and conclusion presented in this paper are those of the European Bioanalysis Forum and do not necessarily reflect the representative affiliation or company's position on the subject.

\section{Financial \& competing interests disclosure}

The authors have no relevant affiliations or financial involvement with any organization or entity with a financial interest in or financial conflict with the subject matter or materials discussed in the manuscript. This includes employment, consultancies, honoraria, stock ownership or options, expert testimony, grants or patents received or pending, or royalties.

No writing assistance was utilized in the production of this manuscript.

\section{References}

1. European Bioanalysis Forum (EBF) Homepage. https://e-b-f.eu/home-2/

2. WHO. Coronavirus disease (COVID-19) pandemic. http://www.who.int/emergencies/diseases/novel-coronavirus-2019

3. European Bioanalysis Forum (EBF). Past Conferences. https://e-b-f.eu/conferences/past-conferences-2/

4. Velghe S, Mercolini L, McDonagh R et al. Feedback from the fourth European Bioanalysis Forum Young Scientist Symposium. Bioanalysis 10(20), 1631-1634 (2018).

5. Protti M, Babylon Y, Delahaye L et al. Feedback from the Fifth European Bioanalysis Forum Young Scientist Symposium. Bioanalysis 11(16), 1453-1458 (2019).

6. Popat R, Adhao V, Thenge $\mathrm{R}$ et al. Green bioanalytical chemistry: a review. JCPR 6(2), 1809-1824 (2016).

7. Howes L. Can laboratories move away from single-use plastic? ACS Cent. Sci. 5(12), 1904-1906 (2019).

8. Olives A, González-Ruiz V, Martín M et al. Sustainable and eco-friendly alternatives for liquid chromatographic analysis. ACS Sustainable Chem. Eng. 5(7), 5618-5634 (2017).

9. Granath B, Jalkesten E, Cotgreave I et al. Strategic focus on 3R principles reveals major reductions in the use of animals in pharmaceutical toxicity. PLoS ONE 9(7), e101638 (2014).

10. Langley G, Farnaud S. Opinion: microdosing: safer clinical trials and fewer animal tests. Bioanalysis 2(3), 393-395 (2010).

11. Google. Year in Search (2020). https://trends.google.com/trends/yis/2020/GLOBAL/?fbclid=IwAR2NmV4og5tViaEV07sxwc513Fd8r wDBojdlZZCUELSzEpND8Ns0rbWPz58 